\title{
Surgical treatment of a spontaneous spinal epidural hematoma under antithrombotic treatment of a multisystem inflammatory syndrome in children associated with SARS-COV-2
}

\author{
Alexander Romagna $^{1,2}$ (D) Fabian Potthast ${ }^{3} \cdot$ Marc-Matthias Steinborn $^{4} \cdot$ Stefan Burdach $^{3} \cdot$ Jens Lehmberg $^{1}$
}

Received: 8 June 2021 / Accepted: 22 September 2021 / Published online: 29 September 2021

(c) The Author(s), under exclusive licence to Springer-Verlag GmbH Germany, part of Springer Nature 2021

\begin{abstract}
Multisystem inflammatory syndrome in children (MIS-C) is a novel syndrome of multisystemic inflammation affecting children. This case report documents an exceptional and severe complication of an epidural hematoma in a 3-year-old boy under the treatment of MIS-C. During the course of the disease, the patient suffered from a hypocoagulable state and an extensive multisegmental epidural hematoma in the cervical spinal canal. This led to severe anterior spinal cord compression and tetraparesis. Extensive emergency surgery had to be carried out to reverse rapid clinical deterioration.
\end{abstract}

Keywords MIS-C $\cdot$ SARS-COV-2 $\cdot$ Epidural hematoma $\cdot$ Cervical spine

\section{Introduction}

We present the case of a 3-year old boy who was initially transferred to the pediatric emergency department with a 5-day history of fever $\left(40^{\circ} \mathrm{C}\right)$, diarrhea, and vomiting. Prior medical history was unremarkable. Extended history revealed that the mother had close contact to a severe acute respiratory syndrome coronavirus type 2 (SARS-CoV-2)-positive person about a month prior, but was tested negative for SARS-CoV-2 thereafter.

\section{Case presentation}

On physical examination, a combination of nuchal rigidity, dehydration, a pleiomorphic rash, and bilateral nonpurulent conjunctivitis was seen. CSF analysis showed

Alexander Romagna

alexander.romagna@muenchen-klinik.de

1 Department of Neurosurgery, München Klinik Schwabing, Munich, Germany

2 Department of Neurosurgery, University Hospital Salzburg, Paracelsus Medical University, Salzburg, Austria

3 Department of Pediatrics, Technische Universität München (TUM) and München Klinik Schwabing, Munich, Germany

4 Department of Diagnostic and Interventional and Pediatric Radiology, München Klinik Schwabing, Munich, Germany

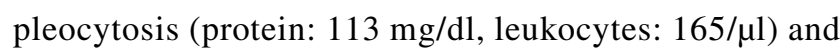
due to rapid clinical deterioration and arterial hypotension the boy was transferred to the pediatric ICU. Under the suspected diagnosis of meningitis a treatment with intravenous cefotaxime, aciclovir, fluid replacement, and vasopressors was initiated. Further CSF analysis showed no evidence for viruses, fungi, or bacteria. Notably, blood tests revealed leukocytosis, elevated inflammation markers and D-dimers, and a status post-SARSCoV-2 infection with SARS-CoV-2-IgG/IgM antibodies (nasopharyngeal SARS-CoV-2 PCR was negative). This disease pattern led to the suspicion of the multisystem inflammatory syndrome in children associated with SARS-CoV-2 (MIS-C) with Kawasaki Disease (KD)-like features [1]. An anti-inflammatory treatment with steroids (prednisolone $2 \mathrm{mg} / \mathrm{kg} / \mathrm{d}$ ), high-dose immunoglobulins $(2 \mathrm{~g} / \mathrm{kg})$, and aspirin ( 3 days with $50 \mathrm{mg} / \mathrm{kg} / \mathrm{d}$, then $5 \mathrm{mg} / \mathrm{kg} / \mathrm{d}$ ) was initiated, as well as a venous thromboembolic prophylaxis with enoxaparin (antifactor Xa level $0.2-0.47$ ) - due to the presumed hypercoagulable state with elevated D-dimers (maximum $7.34 \mathrm{mg} / \mathrm{l}$ ) associated with MIS-C. Under this therapy the arterial hypotension and laboratory parameters continuously improved with the exception of a transient thrombocytopenia (minimum $33 / \mathrm{nl}$ ). Heparine-induced thrombocytopenia (HIT) type 2 was ruled out by serological testing. The patient complained about recurrent neck pain from the day of admission; 10 days after admission the child was suddenly 
Fig. 1 (A) Sagittal T2-weighted magnetic resonance imaging of the cervical spine demonstrating multisegmental anterior epidural hematoma with displacement and compression of the spinal cord. Corresponding intraoperative ultrasound image (B). On intraoperative ultrasound (sagittal plane), the high amount of compression on the spinal cord (upper arrow) by the anterior hematoma (lower arrow) is clearly visible
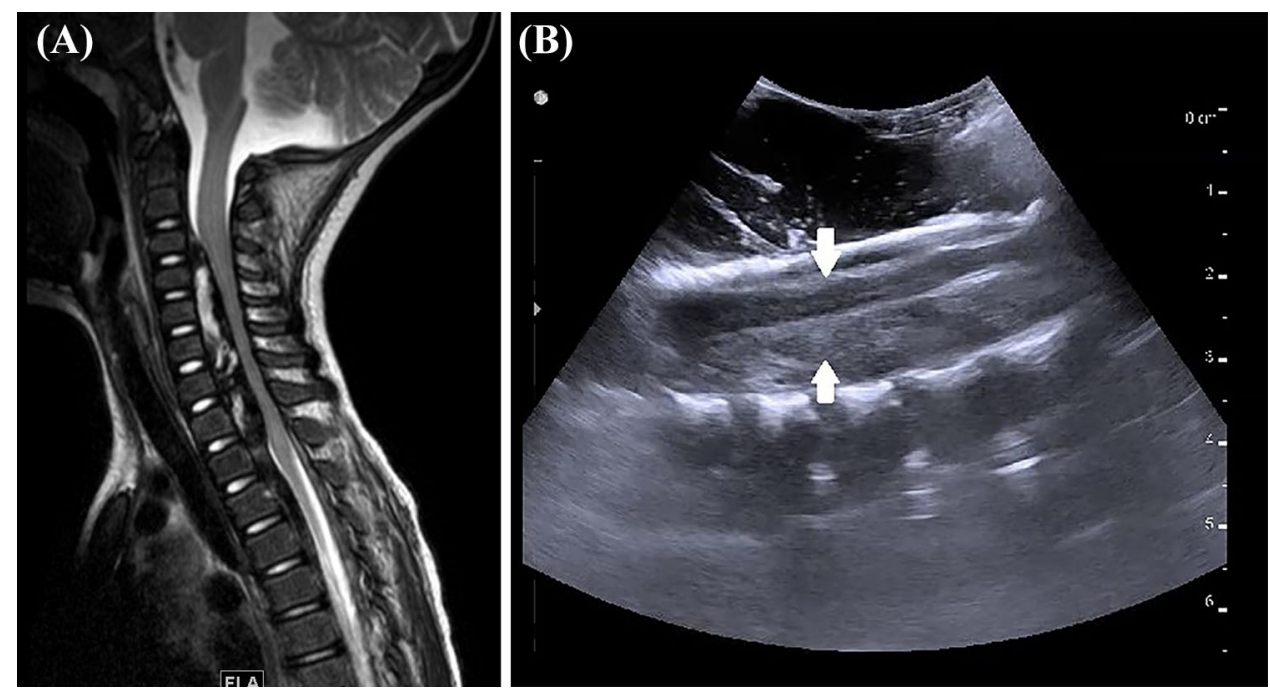

found to have a loss of strength in his right hand. An emergency cranial MRI did not reveal any pathology and the weakness disappeared within 1 day. However, 2 days later the boy developed a rapidly progressive tetraparesis and an MRI of the brain and the spinal canal revealed an extensive multisegmental epidural hematoma in the cervical spinal canal leading to severe anterior spinal cord compression in C3-7 (Fig. 1A, B).

\section{Surgical procedure}

Emergency surgery was initiated within $12 \mathrm{~h}$ after onset of symptoms. In a first posterior procedure, a multilevel laminoplasty failed to achieve a sufficient decompression: the hematoma appeared organized and firmly adherent to the anterior aspect of the cervical spinal cord (Fig. 1A, B). Since only minimal clinical benefit was seen after surgery, a second procedure with an extensive laminectomy $\underline{\mathrm{C} 3-\mathrm{Th} 3}$ was performed. The osseous decompression was further extended bilaterally and the organized hematoma was microsurgically removed under continuous irrigation. Re-insertion of the cervical laminae was deemed to bear the risk of additional cord compression; hence, only the laminae Th2 and Th3 were re-inserted and fixed with microplates. Post-operative spinal MRI and CT scan confirmed a status post-hematoma removal with sufficient decompression of the cervical cord and bony structures (Fig. 2A, B). Clinically, the child improved with right-sided weakness dominating over his left-sided weakness. Overall, his functional outcome changed from grade $\mathrm{B}$ to grade $\mathrm{C}$ on the American Spinal Injury Association impairment scale. Ultimately, he was discharged 28 days after admission and referred to a comprehensive pediatric rehabilitation facility. Three months after surgery, only a mild residual ataxia with otherwise good muscle strength and functional performance was seen.

\section{Discussion}

Children generally suffer from milder forms of COVID-19 (coronavirus disease 2019) than adults [2]. This is explained by the lack of underlying conditions as seen in adults, as well
Fig. 2 (A) Post-operative sagittal T2-weighted magnetic resonance imaging of the cervical spine after multilevel laminectomy and removal of large parts of the hematoma. No obvious signal alterations of the formerly compressed spinal cord. (B) Sagittal reconstruction of a CT scan demonstrates the extensive bilateral osseous defects after decompression
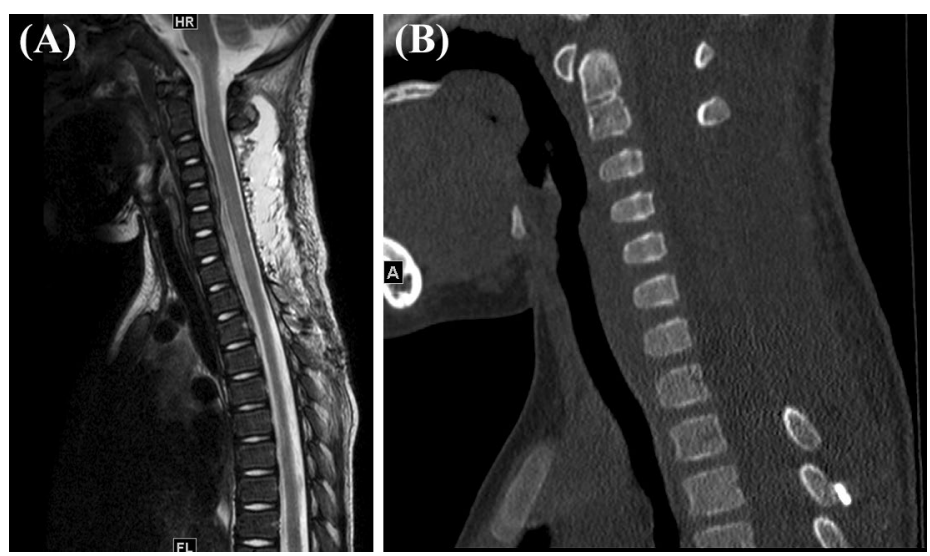
as the fact that the immune systems of children and adults are different $[2,3]$. An exception is the novel syndrome MIS$\mathrm{C}$, defined by the WHO as a syndrome with several clinical and laboratory features and evidence of COVID-19, or likely contact with a person currently or formerly affected by COVID-19 [1].

In the presented case, blood tests revealed a status postSARS-CoV-2 infection with SARS-CoV-2-IgG/IgM antibodies although nasopharyngeal SARS-CoV-2 PCR tests were negative. This led to the suspicion that the patient must have had contact with SARS-CoV-2 in the past. The limited studies on false-negative diagnostic COVID tests indicate a frequency of $20 \%$. Hence, false-negative PCR testing might have provided false reassurance in our case too. Eventually, we believe that a large-scale autoimmune response was triggered as described in numerous MIS-C case series lately all over the globe [4-7]. The true epidemiological impact remains to be determined. In this report, we present an unusual course of this novel condition with pitfalls to consider: (i) during the hyperinflammatory and hypercoagulable period this patient with MIS-C and KDlike features received a antiplatelet and anticoagulation therapy with aspirin and enoxaparin. This is in accordance with guidelines and based on the risk of venous and arterial thromboembolism [7, 8]. The rapidly evolving thrombocytopenia, however, might have shifted towards a hypocoagulable state leading to the subsequent intraspinal hemorrhage. (ii) MR-imaging of the spine was indicated in this case in spite of the fact that spontaneous spinal epidural hematomas are rare in pediatric patients. (iii) Regarding the surgical strategy: the space-occupying epidural hematoma was located anterior to the cervical spinal cord. Given the age of the child, a posterior approach was chosen instead of an anterior approach, i.e., multilevel corpectomy. This is in line with the literature $[9,10]$. In an effort to minimize the risk for post-operative kyphosis, an aggressive facetectomy was avoided and preservation of the C2 lamina was deemed necessary for the prevention of late cervical swan-neck deformity (Fig. 2A, B). With regard to the sagittal balance, no preoperative loss of lordosis was seen. Still, risk factors such as young age (i.e., incomplete cervical spine maturation) and removal of more than four laminae could naturally might lead to post-laminectomy kyphosis since a less debilitating laminoplasty previously failed $[11,12]$. From a technical perspective, recent reports point to hemilaminectomy and laminectomy in spontaneous spinal epidural hematomas $[9,10]$. A "wash-and-go" technique via catheter irrigation as previously described was not possible due to the prolonged nature of the hematoma $[10,13]$. Thus, the initial multilevel laminoplasty could probably have been avoided due to the moderate decompressive effect. Ultimately, the sensorimotor deficits improved after surgery.
These aspects are in line with the literature outlining the importance of early surgery for neurologic recovery [9]. Still, it has also been shown that complete recovery can be seen after late presentations. Therefore, we advocate surgery even in children with delayed presentation and neurologic deficits in stable hemostasis.

This pediatric case illustrates the exceptional and severe complication of an epidural hematoma in a young boy under the treatment of MIS-C.

The clinical recovery after extensive decompression outlines the importance of early diagnosis and extensive decompressive surgery especially if severe sensorimotor deficits are seen. Ultimately, understanding the post-COVID-19 mosaic and optimizing its management represents an unprecedented challenge, especially in pediatrics.

\section{Declarations}

Conflict of interest On behalf of all authors, the corresponding author states that there is no conflict of interest.

\section{References}

1. WHO (2020) Multisystem inflammatory syndrome in children and adolescents temporally related to COVID-19. Scientific Brief. 12 February 2021. https://www.who.int/news-room/ commentaries/detail/multisystem-inflammatory-syndrome-inchildren-and-adolescents-with-covid-19

2. Brodin P (2020) Why is COVID-19 so mild in children? Acta Paediatr 109:1082-1083. https://doi.org/10.1111/apa.15271

3. Simon AK, Hollander GA, McMichael A (2015) Evolution of the immune system in humans from infancy to old age. Proc Biol Sci 282:20143085. https://doi.org/10.1098/rspb.2014.3085

4. Kucirka LM, Lauer SA, Laeyendecker O, Boon D, Lessler J (2020) Variation in false-negative rate of reverse transcriptase polymerase chain reaction-based SARS-CoV-2 tests by time since exposure. Ann Intern Med 173:262-267. https://doi.org/10.7326/ M20-1495

5. Webb K, Abraham DR, Faleye A, McCulloch M, Rabie H, Scott C, Cape Town MISC-Team (2020) Multisystem inflammatory syndrome in children in South Africa. Lancet Child Adolesc Health 4:e38. https://doi.org/10.1016/S2352-4642(20)30272-8

6. Davies P, Evans C, Kanthimathinathan HK et al. (2020) Intensive care admissions of children with paediatric inflammatory multisystem syndrome temporally associated with SARS-CoV-2 (PIMS-TS) in the UK: a multicentre observational study. Lancet Child Adolesc Health 4:669-677. https://doi.org/10.1016/S23524642(20)30215-7

7. Feldstein LR, Rose EB, Horwitz SM et al. (2020) Multisystem inflammatory syndrome in U.S. children and adolescents. N Eng1 J Med, NEJMoa2021680. https://doi.org/10.1056/ NEJMoa2021680

8. Faganel Kotnik B, Zajc Avramovič M, Kitanovski L, Avčin T (2021) Feb 14 (2021) Prophylactic anticoagulation: comment on the American College of Rheumatology Guidance for Management of Multisystem Inflammatory Syndrome in Children Arthritis Rheumatol. https://doi.org/10.1002/art.41690

9. Kim JK, Kim TH, Park SK, Hwang YS, Shin HS, Shin JJ (2013) Acute spontaneous cervical epidural hematoma mimicking 
cerebral stroke: a case report and literature review. Korean J Spine 10:170-173. https://doi.org/10.14245/kjs.2013.10.3.170

10. Aycan A, Ozdemir S, Arslan H, Gonullu E, Bozkına C (2016) Idiopathic thoracic spontaneous spinal epidural hematoma. Case Rep Surg 2016:5430708. https://doi.org/10.1155/2016/5430708

11. Babayev R, Ekşi MŞ (2016) Spontaneous thoracic epidural hematoma: a case report and literature review. Childs Nerv Syst 32:181-187. https://doi.org/10.1007/s00381-015-2768-5

12. Da Silva AJF, Lopes FAC, Mendes WR (2020) Multiple-level cervical spine trauma in children: case report and literature review.
Trauma Case Rep 27:100298. https://doi.org/10.1016/j.tcr.2020. 100298

13. Tanriverdi O, Gungor A, Coban MK, Okay O, Kamaci U (2017) A new less invasive technique for multiple-level spontaneous spinal epidural hematomas: wash-and-go technique. J Neurol Surg A Cent Eur Neurosurg 78:198-201. https://doi.org/10. 1055/s-0036-158243

Publisher's Note Springer Nature remains neutral with regard to jurisdictional claims in published maps and institutional affiliations. 